\title{
Electron Capture Reactions and $\beta$-Decays in Astrophysical Processes
}

\section{Toshio Suzuki*}

Department of Physics, College of Humanities and Sciences, Nihon University

National Astronomical Observatory of Japan

E-mail: suzukiephys.chs.nihon-u.ac.jp

Michio Honma

Center for Mathematical Sciences, University of Aizu

\section{Takashi Yoshida}

Department of Astronomy, Graduate School of Science, University of Tokyo

\section{Toshitaka Kajino}

National Astronomical Observatory of Japan

Department of Astronomy, Graduate School of Science, University of Tokyo

\section{Takaharu Otsuka}

Department of Physics and Center for Nuclear Study, University of Tokyo National Superconducting Cyclotron Laboratory, Michigan State University

Gamow-Teller (GT) strengths in Ni and Co isotopes are studied by shell model calculations with the use of a new Hamiltonian in $f p$-shell, GXPF1J. The GT strengths obtained are used to evaluate electron capture reaction rates at stellar environments. The calculated capture rates in ${ }^{56} \mathrm{Ni},{ }^{58} \mathrm{Ni}$ and ${ }^{60} \mathrm{Ni}$ as well as ${ }^{55} \mathrm{Ni}$ are found to reproduce well the rates obtained by using the experimental GT strengths. The evaluation of the capture rates has been considerably improved compared with previous calculations.

$\beta$-decay half-lives for waiting-point nuclei at $N=126$ are studied by shell-model calculations including both the GT and first-forbidden (FF) transitions. The FF transitions reduce the halflives obtained with the GT contributions by nearly twice to several times. The half-lives obtained here are short compared to standard values of FRDM. Possible implications of the short half-lives on the r-process nucleosynthesis are investigated. Quenching of axial-vector $\left(g_{A}\right)$ and vector $\left(g_{V}\right)$ coupling constants at and near the waiting-point nuclei is discussed.

XII International Symposium on Nuclei in the Cosmos,

August 5-12, 2012

Cairns, Australia

\footnotetext{
*Speaker.
} 


\section{Gamow-Teller Strengths in Ni and Co Isotopes and Electron Capture Rates}

Electron capture reactions play the most essential roles in the core-collapse processes at the last stage of the life-cycle of stars. Accurate evaluations of the electron capture rates at high densities and temperatures are quite important to determine the initial conditions for the nucleosynthesis in supernova explosions.

Gamow-Teller (GT) transition strengths in $\mathrm{Ni}$ and Co isotopes are studied by shell model calculations with the use of a new Hamiltonian in $f p$-shell, GXPF1J[1], which can successfully describe spin-dependent transitions in $f p$-shell, for example, GT strength in ${ }^{58} \mathrm{Ni}$ and $\mathrm{M} 1$ strengths in $f p$-shell nuclei. The GT strengths for GXPF1J are generally more fragmented compared to those of conventional Hamiltonians such as KB3G[2] as shown in Fig. 1(a) for ${ }^{56} \mathrm{Ni}$. The GT strength by GXPF1J shows a two-peak structure while KB3G gives only a single peak. Recently, the two-peak structure of the GT strength was confirmed by $(p, n)$ experiment[3]. One of the reasons of the different structure of the GT strength can be attributed to a wider single-particle shell gap between $0 f_{7 / 2}$ and $0 f_{5 / 2}$ orbits for GXPF1J. The GT strength in ${ }^{55}$ Co recently measured by $(\mathrm{p}, \mathrm{n})$ reaction is also found to be reproduced well by GXPF1J[ $₫$, which is more fragmented compared to the case of KB3G as shown in Fig. 2(a).
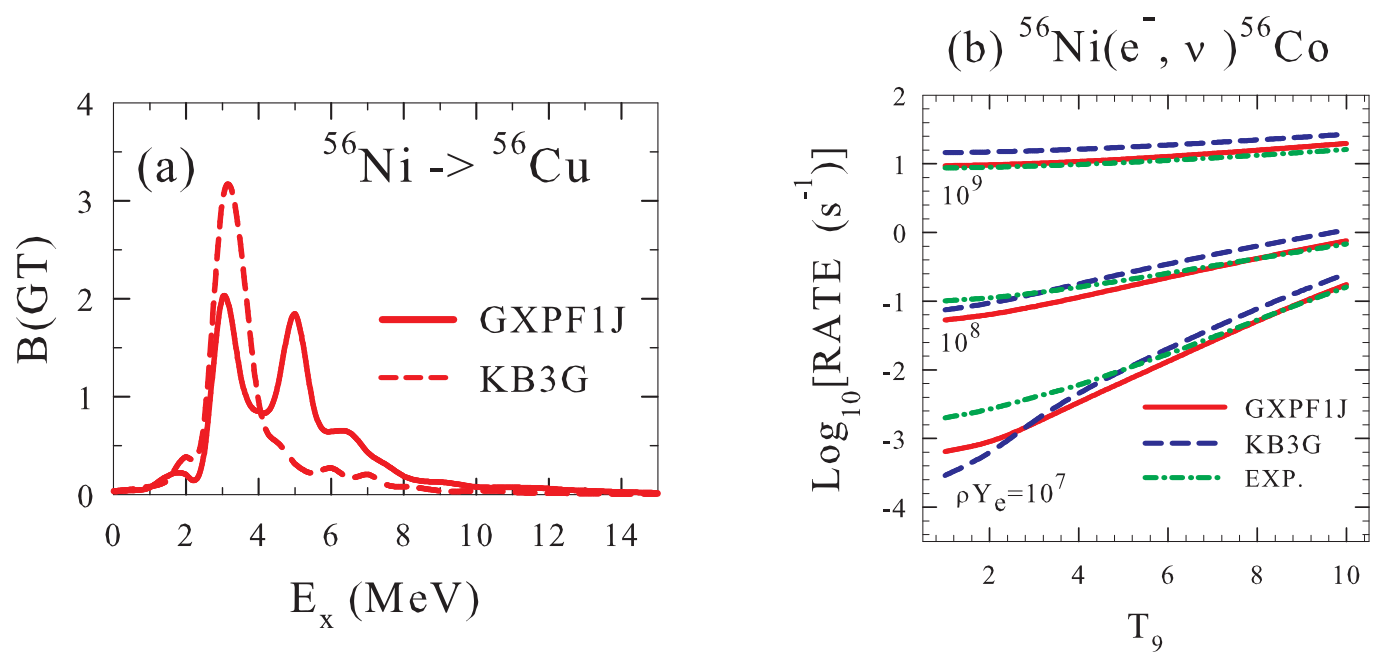

Figure 1: (a) The GT strength in ${ }^{56} \mathrm{Ni}$ obtained by GXPF1J and KB3G. (b) Electron capture rates on ${ }^{56} \mathrm{Ni}$ evaluated by GXPF1J, KB3G and experimental GT strength.

The GT strengths obtained are used to evaluate electron capture rates at stellar environments[[]]. The capture rates at high densities $\rho Y=10^{7-9} \mathrm{~g} / \mathrm{cm}^{3}$ and high temperatures $T=T_{9} \times 10^{9} \mathrm{~K}$ are shown in Fig. 1(b) for ${ }^{56} \mathrm{Ni}$.

The capture rates are calculated by

$$
\lambda=\frac{\ln 2}{6146(s)} \sum_{i} W_{i} \sum_{f} B(G T ; i \rightarrow f) \int_{\omega_{\min }}^{\infty} \omega p\left(Q_{i f}+\omega\right)^{2} F(Z, \omega) S_{e}(\omega) d \omega
$$




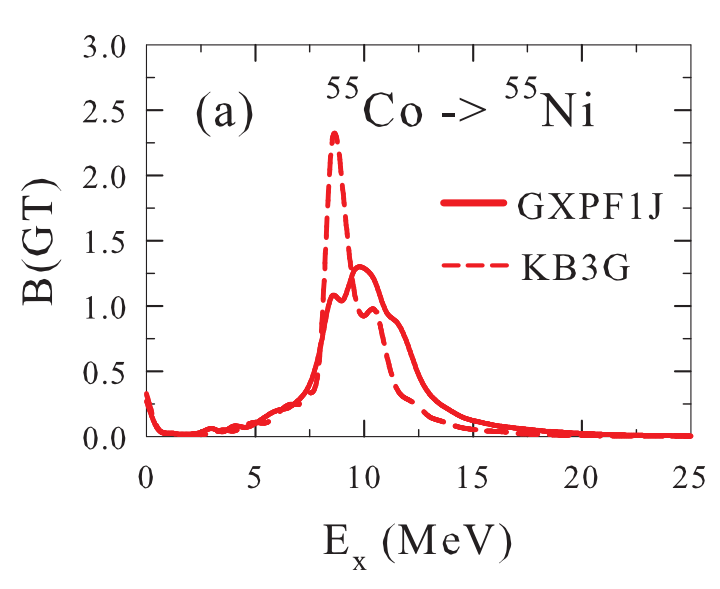

(b) ${ }^{55} \mathrm{Ni}\left(\mathrm{e}^{-}, \mathrm{v}\right){ }^{55} \mathrm{Co}$

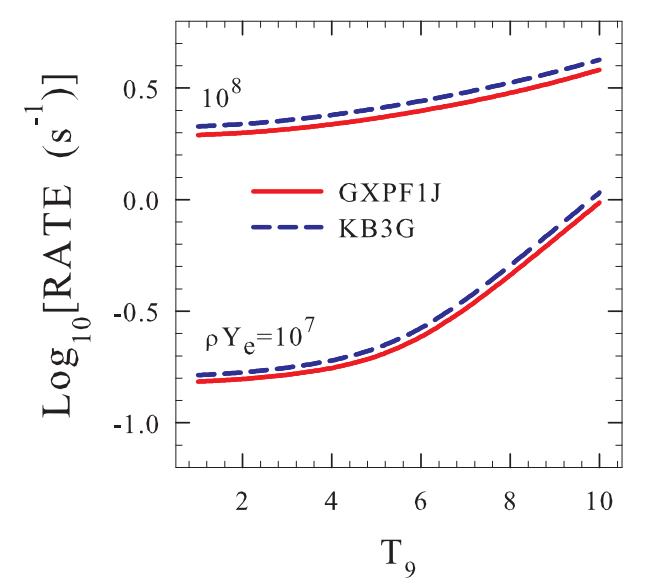

Figure 2: (a) The GT strength in ${ }^{55} \mathrm{CO}$ obtained by GXPF1J and KB3G. (b) Electron capture rates on ${ }^{55} \mathrm{Ni}$ evaluated by GXPF1Jand KB3G.

where $\omega$ and $p$ are electron energy and momentum in units of $m_{e} c^{2}$ and $M_{p}$, respectively. $Q_{i f}$ is the $Q$-value for the reaction, and $W_{i}$ is the statistical weight facor for the initial state. $F(Z, \omega)$ is the Fermi function, and $S_{e}(\omega)$ is the Fermi-Dirac distribution for electrons where the chemical potential, $\mu_{e}$, is determined from the density, $\rho Y_{e}$, by

$$
\rho Y_{e}=\frac{1}{\pi^{2} N_{A}}\left(\frac{m_{e} c}{\hbar}\right)^{3} \int_{0}^{\infty}\left(S_{e}-S_{p}\right) p^{2} d p
$$

where $N_{A}$ is the Avogadro constant and $S_{p}$ is the Fermi-Dirac distribution for positrons with the chemical potential $\mu_{p}=-\mu_{e}$. It can become as large as $5 \sim 10 \mathrm{MeV}$ at high densities $\rho Y_{e}=10^{9} \sim$ $10^{10} \mathrm{~g} / \mathrm{cm}^{3}$. The reaction rates become larger at higher densities because of the large chemical potential of electrons.

We find that GXPF1J reproduces well the capture rates in ${ }^{56} \mathrm{Ni}$ obtained by the experimental GT strength at higher densities and temperatures. The difference of the capture rates in ${ }^{55} \mathrm{Ni}$ between GXPF1J and KB3G is shown in Fig. 2(b). The capture rates for GXPF1J are smaller than those for $\mathrm{KB} 3 \mathrm{G}$ due to larger spreading of the strength.

The calculated capture rates in ${ }^{58} \mathrm{Ni}$ and ${ }^{60} \mathrm{Ni}$ are also found to reproduce well the rates obtained by using the experimental GT strengths [6, 7]. Accurate evaluation of the electron capture rates in $\mathrm{Ni}$ and $\mathrm{Co}$ isotopes has been achieved by using GXPF1J compared with previous calculations[8].

\section{2. $\beta$-Decay Half-Lives at and near $N=126$ Waiting Point Nuclei and R-Process Nucleosynthesis}

$\beta$-decay half-lives at the waiting point nuclei with $N=126$ are evaluated by shell-model calculations including contributions from both the GT and FF transitions[9]. A modified G-matrix, 
which describes well the energy spectra of the isotones with $Z=77 \sim 80[10]$, is used for the shellmodel interaction. The quenching of $g_{A}, g_{A} / g_{A}^{\text {free }}=0.70$, is adopted for both the GT and FF transitions except for $0^{-}$, where an enhancement of the $\vec{\sigma} \cdot \vec{p}$ term due to the meson-exchange current effects are taken into account.

The decay rates, $\Lambda$, as well as the partial half-lives, $t_{1 / 2}$, of the transitions are obtained by the following formulae, 11, 12, 13,

$$
\begin{array}{r}
\Lambda\left(s^{-1}\right)=\ln 2 / t_{1 / 2}=f / 8896(s) \\
f=\int_{1}^{w_{0}} C(w) F(Z, w) p w\left(w_{0}-w\right)^{2} d w \\
C(w)=K_{0}+K_{1} w+K_{-1} / w+K_{2} w^{2},
\end{array}
$$

where $w$ the electron energy, $F(Z, w)$ is the Fermi function, and $K_{n}$ 's depend on nuclear transition matrix elements. Here, relativistic corrections from the expansion of electron radial wave functions in powers of electron mass and nuclear charge parameters are included; matrix elements of onebody operators, $[\vec{r} \times \vec{\sigma}]^{\lambda}$ with $\lambda=0,1,2$ and $\vec{r}$, as well as those from weak hadronic currents, $\gamma_{5}$, $\vec{\alpha}$, are taken into account for the FF transitions. The effects of finite nuclear charge distribution are taken into account [11, 12]. The so-called $\xi$-approximation was not made.

Calculated half-lives are shown in Fig. 3 (left) for GT and GT+FF cases as well as those of FRDM[14] and CQRPA[15]. The FF transitions reduce the half-lives by about 1.5 to several times compared to the case of GT only. The net half-lives are found to be short compared with those of FRDM model[14], which are often used as standard values for astrophysical calculations. They are, on the other hand, longer than those of CQRPA 15. We also note that the odd-even staggering seen in FRDM is not found in the present shell-model results.

Effects of the short half-lives obtained here on r-process nucleosynthesis are investigated. The dependence of the abundances of the elements around mass number $A \sim 195$ on the half-lives of the isotones is studied for various astrophysical conditions. An analytic model of neutrino-driven winds [16] is used for the time evolution of thermal profiles (see Ref. [9] for more details). The neutrino energy spectra are assumed to obey Fermi distributions with zero chemical potentials. The temperatures of of $v_{e}, \bar{v}_{e}$ and $v_{x}=v_{\mu, \tau}, \bar{v}_{\mu, \tau}$ are set to be $\left(T_{v_{e}}, T_{\bar{v}_{e}}, T_{v_{x}}\right)=(3.2 \mathrm{MeV}, 5 \mathrm{MeV}, 6$ $\mathrm{MeV}$ )[17]. The abundances of elements in the r-process nucleosynthesis obtained by using the present $\beta$-decay half-lives for $N=126$ isotones are compared with those of the standard FRDM half-lives in Fig. 3 (right). The third peak of the element abundances is found to be slightly shifted toward higher mass region. This shift is a robust effect independent of the present astrophysical condition for the r-process.

We now discuss the dependence of the $\beta$-decay half-lives on the quenching factors of $g_{A}$ and $g_{V}$. A large quenching of both $g_{A}$ and $g_{V}$ for $1^{-}$spin-dipole transitions was suggested by FF $\beta$ decays in the lead region; $\left(g_{A} / g_{A}^{\text {free }}, g_{V} / g_{V}^{\text {free }}\right)=(0.47,0.64)$ [18]. Two sets of quenching factors, $(0.34,0.67)$ and $(0.51,0.30)$, are obtained from the analysis of ${ }^{205} \mathrm{Tl}\left(1 / 2^{+}\right) \rightarrow{ }^{205} \mathrm{~Pb}\left(1 / 2^{-}\right)[10]$. A large quenching of $g_{A}$ is suggested from the studies of FF $\beta$-decays of the isotones with $Z=78 \sim 80$, while a large quenching for $g_{V}$ is unlikely (see Ref. [9]).

Dependence of the calculated half-lives on the quenching of $g_{A}$ and $g_{V}$ is studied for the $N=126$ isotones. Half-lives obtained with the set $(0.34,0.67)$ for the $1^{-}$transitions are shown by 

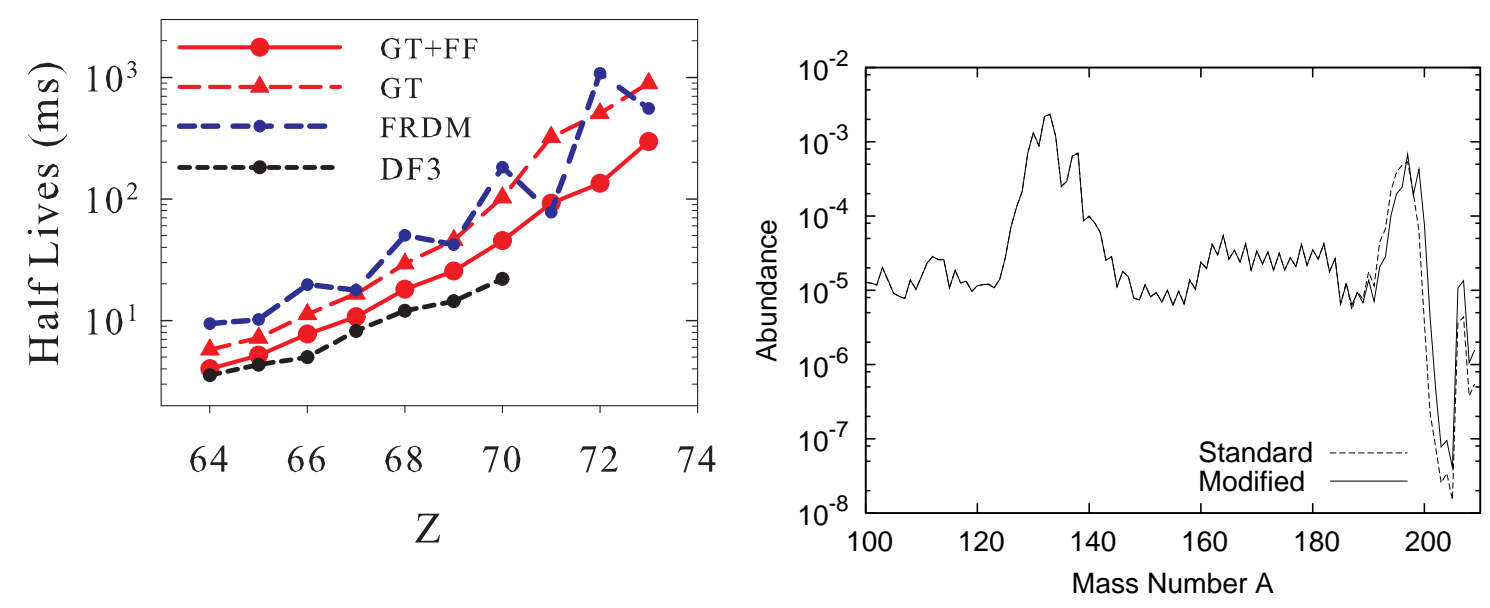

Figure 3: Calculated $\beta$-decay half-lives for isotones with $N=126$ obtained with GT and GT+FF transitions. Half-lives for FRDM[14] and CQRPA[15] (denoted as DF3) are also shown (left). Element abundances of r-process nucleosynthesis obtained with the present shell-model half-lives (denoted as "Modified") as well as those of the FRDM model (denoted as "Standard") (right).

long-dashed line in Fig. 4(a). The half-lives get longer but still remain shorter than those of Ref. [14] except for $Z=71$. The dependence of the half-lives on the $Q$-values of the transitions are also studied. The present shell-model calculations give smaller $Q$-values compared to the experimental ones for $Z=78 \sim 80$, while there is no experimental information on the $Q$-values for $Z=64 \sim 73$. When calculated $Q$-values are increased by $1 \mathrm{MeV}$ for $Z=64 \sim 73$, the half-lives become small as shown by short-dashed line in Fig. 4(a). The effects are found to be rather large.

Dependence of the FF $\beta$-decay half-lives on the quenching factors is also studied in ${ }^{204} \mathrm{Pt}$ and some nuclei near the waiting points, ${ }^{203} \mathrm{Pt},{ }^{202} \mathrm{Ir}$ and ${ }^{201} \mathrm{Ir}$. Recently, experimental information on the half-lives of these nuclei become available. The half-lives of FRDM[14] are far off the experimental values[19, 20]. A set of the quenching $\left(g_{A} / g_{A}^{\text {free }}, g_{V} / g_{V}^{\text {free }}\right)=(0.70,1.0)$ is rather consistent with the experimental values except for ${ }^{201}$ Ir as shown in Fig. 4(b). A large quenching of $g_{V}$ is not favored for the Pt isotopes similar to the case of the $N=126$ isotones with $Z=78 \sim 80$. Quenching of $g_{V}$ is not taken for the case of DF3-QRPA[15] also. Further study is necessary to understand the quenching of $g_{A}$ and $g_{V}$ in nuclei around this region.

\section{References}

[1] M. Honma et al., Phys. Rev. C 65, 061301 (2002); ibid. C 69, 034335 (2004); Journal of Physics: Conference Series 20, 7 (2005).

[2] E. Caurier et al., Rev. Mod. Phys. 77, 427 (2005).

[3] M. Sasano et al., Phys. Rev. Lett. 107, 202501 (2011).

[4] M. Sasano et al., Phys. Rev. C, in press.

[5] T. Suzuki, M. Honma, H. Mao, T. Otsuka and T. Kajino, Phys. Rev. C 83, 044619 (2011). 

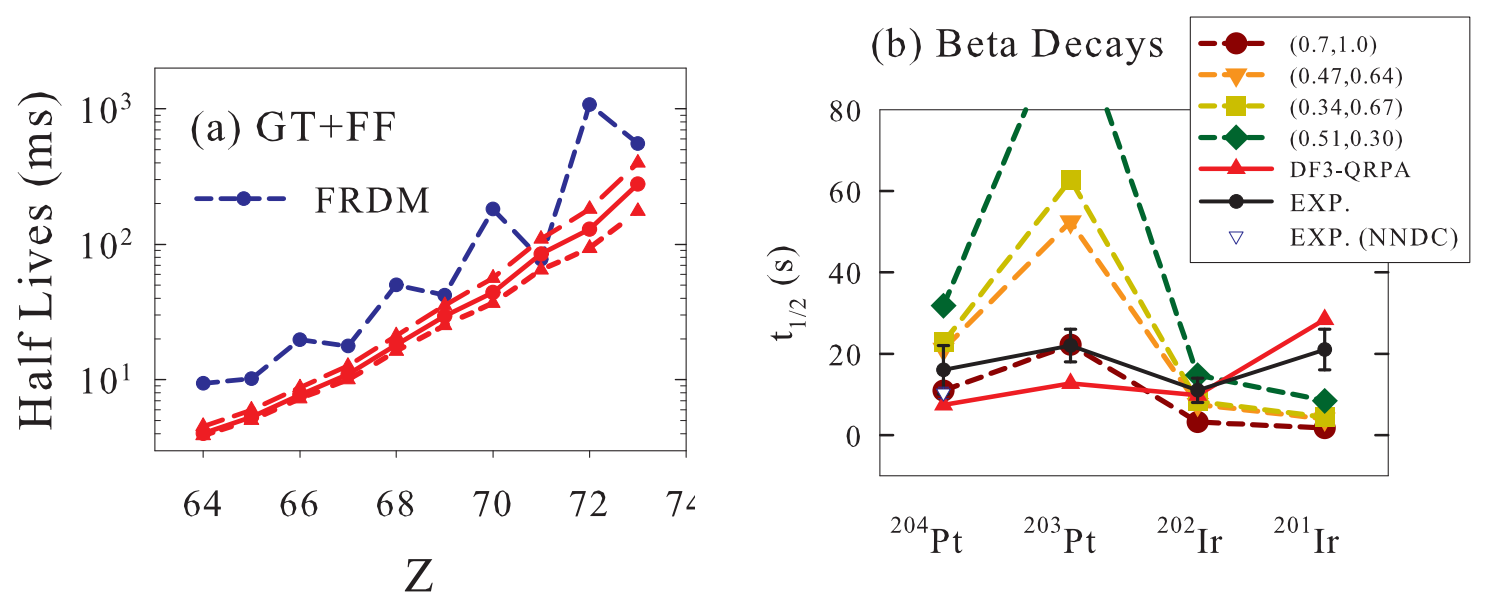

Figure 4: (a) The same as for Fig. 3 (left). Half-lives with larger quenchings in $g_{A}$ and $g_{V}$ are shown by long-dashed line. $Q$-value is enhanced by $1 \mathrm{MeV}$ for short-dashed line. (b) Dependence of $\beta$ half-lives of Pt and Ir isotopes on the quenching factors of $g_{A}$ and $g_{V}$. Experimental values are taken from Refs. [19] and [20].

[6] M. Hagemann et al., Phys. Lett. B579, 251 (2004).

[7] N. Anantaraman et al., Phys. Rev. C 78, 065803 (2008).

[8] K. Langanke and G. Martinez-Pinedo, Nucl. Phys. A673, 481 (2000).

[9] T. Suzuki, T. Yoshida, T. Kajino and T. Otsuka, Phys. Rev. C 85, 015802 (2012).

[10] S. J. Steer et al., Phys. Rev. C 78, 061302 (2008);

L. Rydström et al., Nucl. Phys. A512, 217 (1990).

[11] E. K. Warburton, J. A. Becker, B. A. Brown and D. J. Millener, Annals of Physics 187, 471 (1988);

H. Behrens and W. Bühring, Nucl. Phys. A162, 111 (1971).

[12] H. Schopper, Weak Interactions and Nuclear Beta Decays (North-Holland, Amsterdam, 1966).

[13] I. S. Towner and J. C. Hardy, Nucl. Phys. A179, 489 (1972).

[14] P. Moller, J. R. Nix and K.-L. Kratz, At. Data Nucl. Data Tables 66, 131 (1997);

P. Moller, B. Pfeiffer and K.-L. Kratz, Phys. Rev. C 67, 055802 (2003).

[15] I. N. Borzov and S. Goriely, Phys. Rev. C 62, 035501 (2000).

[16] K. Takahashi and H.-Th. Janka, in Origin of Matter and Evolution of Galaxies, eds. T. Kajino, S. Kubono, and Y. Yoshii (Singapore: World Scientific), 213 (1997).

[17] T. Yoshida, M. Terasawa, T. Kajino, and K. Sumiyoshi, Astrophys. J. 600, 204 (2004).

[18] E. K. Warburton, Phys. Rev. C 42, 2479 (1990).

[19] J. Benlliure et al., ARIS 2011.

[20] National Nuclear Data Center on-line retrieval system, http://www.nndc.bnl.gov. 